\title{
Tecnologias de interface humano-computacional: realidade virtual e novos caminhos para pesquisa
}

\section{Human-computational interfaces technologies: virtual reality and new roads of research}

\author{
Guilherme Welter WendT ${ }^{1}$ \\ 1 Universidade do Vale do Rio dos Sinos (Unisinos).
}

Recebido: 16/3/2011 - Aceito: 4/4/2011

Wendt GW / Rev Psiq Clín. 2011;38(5):211-2
A realidade virtual (RV) vem sendo empregada nos mais variados contextos e tem sua eficácia comprovada na área da saúde desde que foi noticiado o primeiro caso de sucesso utilizando a tecnologia com propósitos experimentais no tratamento de fobias. Dez anos após, é possível observar o uso da RV em um contexto amplo na saúde mental, como em tratamento de transtornos de ansiedade, anorexia e bulimia nervosa, apoio a diagnóstico e assessoramento psicoterapêutico ${ }^{1}$.

Esse novo paradigma é tributário das profundas transformações sociais e tecnológicas observadas na última década, da disseminação de um novo espaço psicossocial, formado por culturas e ciberculturas e sua consequente influência ao comportamento e à interação humana $^{2}$. Na RV, os usuários interagem em ambiente 3D simulado por computador, utilizam capacetes especiais, equipados com displays de LCD e fones de ouvido para criar um contexto multissensorial.

No Brasil, uma rápida consulta às principais bases de dados (SciELO, Pepsic, Banco de Teses e Dissertações da Capes) permite inferir que o tema é particularmente recente na academia nacional, com escassos estudos veiculados em periódicos. Mesmo na Europa, estudos apontam que recentemente os governantes têm se mobilizado e mensurado o impacto social e econômico da saúde mental, com vistas a enfrentar o desafio e aprimorar técnicas que sejam acessíveis e eficientes à população.

A RV é um procedimento seguro e eficaz na melhora da dor e da ansiedade experimentadas em vários níveis de percepção apontados pelos pacientes ${ }^{3,4}$; recentemente, foram registrados benefícios analgésicos por meio da interação em RV. Dessa forma, o presente artigo busca delinear um breve panorama das aplicações da tecnologia na clínica e em psicologia experimental, com o foco na divulgação de novos horizontes metodológicos e possibilidades de investigação de fenômenos psicológicos.

Nos Estados Unidos, Canadá, Itália, Bélgica e outros países que desenvolveram aplicativos de RV, observa-se o uso da tecnologia empregado até mesmo para o treinamento de profissionais. Esses experimentos simulam, por exemplo, a interação entre o médico e o paciente, reproduzem o cenário de uma sala de emergência lotada, forçando os participantes a construírem e testarem hipóteses no mundo imaginado, aumentando a probabilidade de atuação mais efetiva na prática cotidiana. A figura abaixo ilustra uma sala de RV.

Os ambientes virtuais são compostos por sistemas de navegação que permitem à pessoa explorar e orientar-se no ambiente, sistemas de interação (engajamento) e, finalmente, sistemas de presença e imersão. Esses são os componentes que refletem a sensação subjetiva de o sujeito de "estar presente" no ambiente simulado, que gera um índice estatístico objetivo, mensurado pela plataforma utilizada, chamado de imersão. "O bloqueio de impressões sensoriais da realidade física é uma parte crucial das experiências mais interessantes nas RV intensivas, pois os sentidos estão imersos no mundo virtual" (p. 95)5.

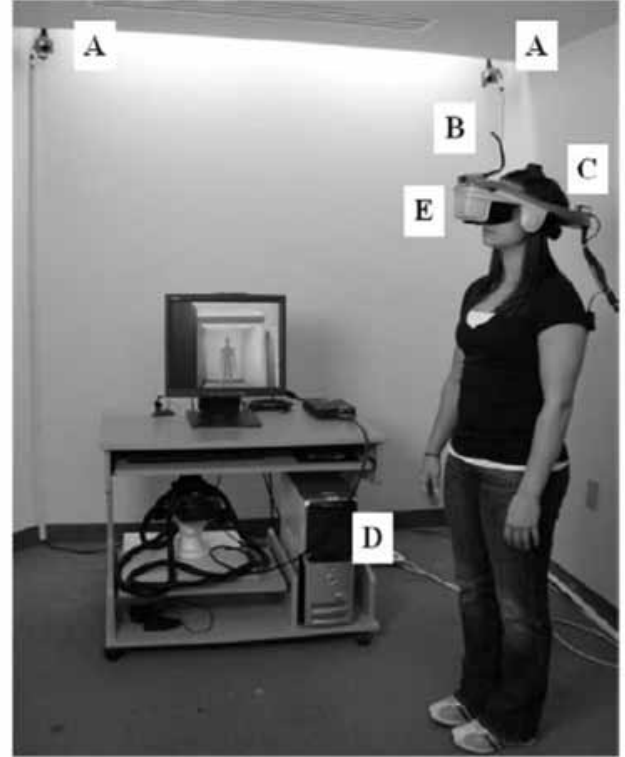

Figura 1. Exemplo de configuração de um ambiente virtual: Câmeras (A), sensor óptico que indica a posição do participante na sala (B), equipamento que reúne informações sobre os movimentos da cabeça do participante (C), que transmite ao computador (D), que determina como o quarto é processado pelo participante e o que ele vê no visor (E) $)^{5}$.

Uma pesquisa veiculada recentemente em um portal de notícias do Brasil relatou o uso da RV para o tratamento de jogo patológico em pacientes espanhóis ${ }^{6}$. Os pesquisadores do Playmancer Project estão empregando tecnologias de RV e desenvolvendo games de saúde, estimulados pelas inúmeras possibilidades de manipulação e experimentação de tecnologias. Além das experiências em jogo patológico, o grupo de pesquisadores já testou e confirmou os benefícios adicionais da RV em tratamentos para bulimia e anorexia nervosa, adições, dislexias síndrome de Asperger, entre outros ${ }^{6}$. No site oficial (www.playmancer.com), há um link com artigos e apresentações científicas que analisam as reações fisiológicas, emocionais e comportamentais de pacientes com distintas patologias ${ }^{6}$.

Estudos de revisão da literatura sugerem que os jogos de computador, em geral, podem servir como uma forma alternativa de tratamento ou uma intervenção adicional em casos como esquizofrenia, asma, reabilitação motora, incentivo à prática de exercícios físicos, emagrecimento, reabilitação neurológica, fobias e psicoeducação ${ }^{7}$. 
Nesse sentido, há uma justificativa bastante atraente para a integração da RV com processos de monitoração fisiológica humana e de imagens. As crianças expostas em um ambiente interativo 3D de distração, em oposição a outras formas de distração, aumentaram significativamente a sua tolerância à dor e o limiar da dor, por exemplo ${ }^{8}$.

Para o diagnóstico psiquiátrico, pesquisas já relataram o uso de RV em grupos de pacientes com transtorno de déficit de atenção e hiperatividade e doença de Alzheimer. Do mesmo modo, estudos indicam que o sucesso terapêutico utilizando RV nos casos de fobia atinge índices de $90 \%{ }^{9}$.

As limitações ao uso da RV nas ciências da saúde dependem somente da imaginação dos pesquisadores ${ }^{9}$. Cabe pontuar, todavia, que a ênfase não deve ser tributada ao uso exclusivo da tecnologia, de modo excludente à psicoterapia. Conforme Castelnuovo et al. ${ }^{1}, \mathrm{o}$ uso da RV deve ser balanceado de acordo com a demanda de cada caso e a RV, como valioso subsídio, deve ser utilizada para a melhora global na qualidade assistencial dispendida.

Além disso, as técnicas de RV parecem aplicáveis a uma amplitude etária significativa de pacientes e pode ser particularmente bem adaptadas para o uso em crianças. O fundamento das práticas com fins de analgesia situa-se no pressuposto de que o ser humano possui limitados recursos atencionais; assim, a distração visa mudar o foco cognitivo do paciente para outro tópico, e não para a sua queixa principal, pois há evidências cognitivas e neurológicas que indicam que imaginar uma ação ou executá-la de fato eliciam ativação cerebral similar.

Jerome e Zaylor ${ }^{10}$ afirmam que a tecnologia emergente alterará perpetuamente o ambiente de cuidados de saúde, mudando continuamente as ferramentas e opções que estão disponíveis para terapeutas. Segundo os mesmos autores, "é importante estudar o impacto dessas mudanças à medida que ocorrem, e é imperativo que as novas competências tecnológicas sejam desenvolvidas com o objetivo de auxiliar os clínicos a integrar essas tecnologias em suas pesquisas e práticas" (p. 478).
A possibilidade de projetar ambientes de estímulo nos quais todas as respostas comportamentais podem ser registradas é um recurso valioso em psicologia experimental, que não está disponível com as técnicas tradicionais. Em virtude da capacidade de coletar informações ao longo do tempo, os cientistas têm a oportunidade, agora, de investigar uma série de fenômenos psicológicos, contribuindo para o acesso a serviços eficazes de saúde e ao desenvolvimento de técnicas específicas para determinadas patologias.

\section{Referências}

1. Castelnuovo G, Gaggioli A, Mantovani F, et al. From psychotherapy to e-therapy: the integration of traditional techniques and new communication tools in clinical settings. Cyberpsychol Behav. 2003;6(4):375-82.

2. Malloy KM, Milling LS. The effectiveness of virtual reality distraction for pain reduction: a systematic review. Clin Psychol Rev. 2010;30:1011-8.

3. Butler AC, Chapman JE, Forman EM, et al. The empirical status of cognitive-behavioral therapy: a review of meta-analyses. Clin Psychol Rev. 2006;26:17-31.

4. Montgomery GH, DuHamel KN, Redd WH. A meta-analysis of hypnotically induced analgesia: how effective is hypnosis? Int J Clin Exp Hypn. 2000;48:138-53.

5. Fox J, Arena D, Bailenson JN. Virtual reality: a survival guide for the social scientist. J Media Psychol. 2009;21(3):95-113.

6. Playmancer.com [Internet]. Grécia: European Serious Gaming 3D Environment, Playmancer Consortium Inc. [updated 2011 Jan 16; cited 2002 Jul 9]. Available from: www.playmancer.com.

7. Gold JI, Belmont KA, Thomas DA. The neurobiology of virtual reality pain attenuation. Cyberpsychol Behavior. 2007;10:536-44.

8. Dahlquist LM, McKenna KD, Jones KK, et al. Active and passive distraction using a head-mounted display helmet: effects on cold pressor pain in children. Health Psychol. 2007;26:794-801.

9. Wiederhold BK, Wiederhold MD. Postface. In: Riva G, Galimberti C. Towards cyberpsychology: mind, cognition, and society in the Internet age. Amsterdan: IOS Press; 2001.

10. Jerome LW, Zaylor C. Cyberspace: creating a therapeutic environment for telehealth applications. Prof Psychol Res Pr. 2000;31:478-83. 